\title{
Reducing Network Cost of Many-to-Many Communication in Unidirectional WDM Rings with Network Coding
}

\author{
Long Long and Ahmed E. Kamal, Senior Member, IEEE
}

\begin{abstract}
In this paper we address the problem of traffic grooming in WDM rings with all-to-all and its generalization to many-to-many service by using network coding. We consider minimizing the number of Line Terminating Equipment (LTE) on two types of unidirectional rings, namely, single-hub and unhubbed rings, as our objective. In single-hub rings, we investigate the minimum cost provisioning of uniform all-to-all traffic in two cases: where network coding is used to linearly combine data, and where it is not used and data is transmitted without coding. We generalize the service mode to many-to-many and evaluate the cost of provisioning. In un-hubbed ring, we propose a multi-hub approach to obtain the minimum cost provisioning in the case of all-to-all and many-to-many traffic. In each type of ring topology, two network scenarios are considered: first, the distinct communication groups in the ring are node-disjoint and second, the different groups may have common member nodes. From our numerical results, we find that under many-to-many traffic pattern for both scenarios, network coding can reduce the network cost by $10-20 \%$ in single-hub rings and $1-5 \%$ in un-hubbed rings in both network scenarios.
\end{abstract}

Index Terms-WDM rings, Traffic grooming, Line Terminating Equipment (LTE), Network coding

\section{INTRODUCTION}

W AVELENGTH-division multiplexing (WDM) technology allows an aggregate traffic on the order of Tbps to be carried on a single fiber, with each wavelength carrying traffic in the tens of Gbps order. However, the traffic demands of network applications are at much smaller granularity than wavelength bit rates. In order to utilize wavelength capacity more efficiently, a number of flows from multiple network connections with sub-wavelength granularity may be packed onto the same wavelength. This process of allocating low bit rate tributary streams to wavelengths with high bandwidth is referred to as traffic grooming. There are two types of traffic grooming problems, static and dynamic. The objective of the static problem is usually to minimize the overall network cost, given the traffic demands, whereas in the dynamic problem, maximizing the throughput or minimizing the blocking probability of connections.

The static traffic grooming problem of unicast traffic has been widely studied in the literature [3], [4], [18]. But recently,

This research was supported in part by grant CNS-0626741 from the National Science Foundation

Long Long and Ahmed E. Kamal are with the Department of Electrical and Computer Engineering, Iowa State University, Ames, IA, USA, 50010 (e-mail: longlong@iastate.edu; kamal@iastate.edu)

Copyright (c) 2009 IEEE. Personal use of this material is permitted. However, permission to use this material for any other purposes must be obtained from the IEEE by sending a request to pubs-permissions@ieee.org. multipoint traffic has become more important in a number of application environments, and this is why a number of studies addressing multipoint traffic grooming have recently appeared in the literature [15], [16], [17]. Among a number of network architectures, ring topologies drew significant attention in the research community due to the availability of legacy SONET equipment [12], [19], [20]. In ring networks, both all-to-all [1], [2], [11], [13], multicast [9], [14] and arbitrary[21] traffic scenarios have been studied. Most of the literature addressing this problem focuses on evaluating and reducing the dominant cost in the optical network, namely, Electronic Add-Drop Multiplexers (ADM), which is required at a node if it either has data to transmit to or receive from another counterpart. The number of ADMs required at a node is only a function of the number of lightpaths established and terminated at the node. Another cost function that is similar to the number of $\mathrm{ADM}$ is the number of e-DaC grooming ports presented in [9], which refers to Electronic Drop-and-Continue grooming ports, in which part of the traffic is dropped off at a node, and the rest of the traffic continues.

In this paper, we address the static traffic grooming problem of a class of multipoint traffic in unidirectional ring networks with the number of electronic Line Terminating Equipments (LTE) ports, be it ADM ports, as the network cost. The number of wavelengths and the cost of other optical equipment, such as the optical splitter (which is negligible compared to the electronic LTEs) are not factors to be considered here. We consider uniform all-to-all traffic grooming, in which all users in the network exchange data. We also consider a generalized case where there are multiple communication groups and each node in a group has to receive the data from all the other users in the same group. A user may belong to multiple groups. We consider two types of unidirectional rings, namely, single-hub and un-hubbed rings. In a single-hub ring [10], all the traffic has to be sent to the hub and then forwarded to the destinations by the hub. In an un-hubbed ring, there is no such hub.

All-to-all traffic can be implemented using two approaches, unicast and multicast. In unicast mode, traffic duplication can only be implemented in the electronic domain, whereas in multicast mode, traffic duplication can be done in the optical domain by using optical splitters. If a node needs to send a traffic stream on two outgoing links, the node requires two LTE ports in unicast mode but only one in multicast mode. All-to-all communication will benefit significantly in terms of the network cost by dividing it into multiple multicast sessions. However, it requires multicast capable nodes to be deployed in 
the network. The corresponding node architecture is referred to as the Tap-and-Continue node, and is introduced in [14], in which a node is capable of replicating optical signals in the optical domain using optical splitters, and one copy is dropped locally, while the remaining signal continues on the fiber without the additional cost of LTEs. Since each node on the unidirectional ring has only one incoming link and one outgoing link, and routing of all lightpaths is fixed along the direction of the ring, there is only one possible way to multicast - drop and forward. We use such node architecture to implement any multicast needed in the network.

Network coding [5] is a promising new technique that enables network nodes to perform algebraic operations on the multiple received packets besides simply forwarding them. It has been applied to a variety of network applications in order to improve the performances, such as in multi-hop wireless networks [28], network tomography [24], network protection [25], [26] and content distribution in peer-to-peer networks [27]. However, it is rarely applied to optical networks for the cost saving objective. We will study two unidirectional ring networks, single-hub and un-hubbed, and investigate whether applying network coding will reduce the network cost to provision given traffic requests.

The paper is structured as follow. In Section II, we introduce network coding and its benefit in saving LTE ports in optical networks. We will explore the network costs with or without applying network coding in single-hub and un-hubbed rings in Section III and IV, respectively. Numerical results of multiple many-to-many communication will be shown in Section V. Finally in Section VI, we conclude the paper.

\section{Network Coding in Optical Network}

Network coding is a novel technique which was originally proposed for improving network capacity, particularly in multicast scenarios[5], [6]. Besides the traditional routing functions, network nodes are designed to linearly combine packets arriving at input edges and transmit those combinations on output ports. By carefully choosing coding coefficients for each coding node in a network, where a coding node refers to a network node that has the capability of forming linear combinations of packets, a network can achieve the maximum multicast throughput for a given multicast request, which is equal to the min-cut max-flow of the network. In other words, given a traffic demand, employing a network coding scheme may provision the traffic by utilizing fewer network resources than the traditional routing scheme. Work has been done in recent years to explore the efficient coding schemes. For example, a classic polynomial time algorithm of codes construction for multicast traffic is proposed in [7] and a random coding scheme is proposed in [8].

In an optical network, traffic flow is carried and conveyed in a lightpath as an optical signal. To establish a lightpath, one LTE port is needed at the source to originate the signal and one is required at the destination to terminate it, and then the total number of LTE ports ${ }^{1}$ required at each node is the total number of lightpaths terminated and originated at this node.

\footnotetext{
${ }^{1}$ In rest of this paper, we use LTE to represent LTE port for short.
}

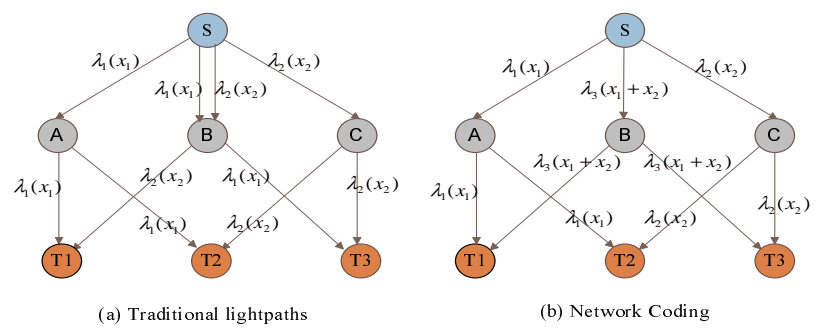

Fig. 1. An example of LTE cost reduction through network coding

Given a multicast service request, if network coding scheme can be employed to reduce the total bandwidth used, the total number of lightpaths required may be reduced, which will consequently result in a saving in the total network cost.

An example is shown in Figure 1 to illustrate the benefits of using network coding to save network cost in terms of LTE in a multicast traffic scenario. In the given network, one multicast session needs to be implemented, in which one source $S$ generates two traffic flows $\lambda_{1}$ and $\lambda_{2}$ and both of them should be received by all three destination nodes $T_{1}, T_{2}$ and $T_{3}$. We also assume that each traffic flow takes an entire wavelength to accommodate. Thus, Figure 1.(a) shows the provisioning of the multicast session by using the traditional routing scheme. In order to achieve the multicast throughput, which is 2 in this case, four lightpaths have to be set up at source $S$ and one of its outgoing links need to carry two traffic flows. In this case, links $(S, A)$ and $(S, C)$ carry one optical signal each and link $(S, B)$ must carry two optical signals. We assume that each intermediate node is deployed with the capability of both multicasting and forwarding functionality in optical domain and thus no LTE is required at node $A, B$ and $C$. Therefore, source node $S$ requires four LTEs to establish the lightpaths and each destination node needs two LTEs to receive the traffic. Network coding is introduced in Figure 1.(b) to provision the same multicast service. Instead of generating four optical signals at source $S$ in (a), only three lightpaths need to be set up as shown, in which two of them carry the original traffic flow $\lambda_{1}$ and $\lambda_{2}$ and the third one carries the encoded data $\lambda_{1}+\lambda_{2}$ generated by performing bitwise operation "Exclusive $O R$ " on the two original signals. In this case, node $T 1$ will receive the traffic $\lambda_{1}$ and $\lambda_{1}+\lambda_{2}$ while node $T 3$ receives $\lambda_{2}$ and $\lambda_{1}+\lambda_{2}$. Clearly, both nodes perform "XOR" operation on the received flow in order to recover the original traffic $\lambda_{2}$ and $\lambda_{1}$, respectively, and hence multicast service is fulfilled. Therefore, employing network coding in the example in Figure 1.(b) reduces the cost by one LTE at the source node $S$ when compared to the cost required in 1.(a).

Although a recent study has introduces ways to conduct the network coding operation in optical domain[22], [23], our scheme is based on the current network infrastructure where network coding can only be implemented in an electronic domain, which requires the traffic that participates in the network coding operation to go through O-E-O conversion at the coding nodes. Such conversion may not be necessary if 
traditional routing is used. Thus, employing network coding may require more LTEs to terminate original traffic and regenerate encoded signals at coding nodes, hence making the problem a trade-off between reduction in the number of coding nodes and the reduction in LTEs in order to use fewer lightpaths in total. Moreover, applying network coding to optical networks also introduces new issues such as where and how should network coding be performed? The answer to this question is straightforward in a single-hub ring network. Since all traffic is collected by the hub, the hub is the perfect node to combine packets. However, it is not as clear in an unhubbed ring. In addition, achieving network coding requires determining the coding scheme and the finite field size $G F(q)$ from which we choose coding coefficients. Of course, network coding does not come for free, and all the coding nodes should be equipped with the capability of coding, which introduces extra computation cost in the application layer. Compared to the cost of LTEs in physical layer, however, such resource consumption is almost negligible. Therefore, we only consider the number of LTEs as the network cost in this paper.

\section{Cost AnALysis in Single-HUb Unidirectional RING}

In this section, we address the problem of grooming all-toall traffic in a single-hub unidirectional ring and then extend the problem to address many-to-many traffic service. In manyto-many communication, multiple groups are required to fulfill the all-to-all service where each group consists of two or more nodes. We consider two different network scenarios where groups are node-disjoint and when a node may belong to different groups. In each scenario, the cost of provisioning traffic is derived using two approaches: traditional routing and by applying network coding. For many-to-many communication, we prove that the traffic grooming problem is NP-complete and also propose an Integer Linear Programming (ILP) formulation to solve the problem optimally in the case where the groups are non-disjoint.

\section{A. Uniform all-to-all traffic}

Under all-to-all service, each node should receive data from all the other nodes on the ring. The problem can be stated as follows: Given a group of $n$ nodes and grooming factor ${ }^{2} g$, each node $i$, generates and transmits traffic at constant rate, $r$, and must receive the traffic sent by other nodes such that the network resources, the LTEs in particular, are minimized.

We assume each data unit has to be transmitted to the hub before being relayed to the destination(s). As mentioned earlier, each node is equipped with optical splitters such that traffic can be duplicated in the optical domain. Moreover, we allow traffic bifurcation, which refers to splitting the traffic from a session over multiple lightpaths, since this may result in the minimum number of lightpaths and achieve the minimum overall cost in the case when $g$ is not a multiple of $r$.

The all-to-all communication process involves two steps. The first step is to deliver traffic upstream from nodes to the

\footnotetext{
${ }^{2}$ Grooming factor refers to the maximum number of low-rate traffic demands that can be multiplexed into one wavelength channel.
}

hub. In the second step, the hub grooms the traffic into the minimum number of wavelengths and multicasts the groomed traffic downstream to every node on the ring. In the upstream direction, each node generates $r$ units of low-rate traffic stream, which requires $\left\lceil\frac{r}{g}\right\rceil$ wavelengths to accommodate the data as well as $\left\lceil\frac{r}{g}\right\rceil$ LTEs to send it, and the hub needs $\left\lceil\frac{r}{g}\right\rceil$ LTEs to receive the traffic from one node. Either grooming the traffic from different nodes before sending it to the hub, or sending data to the hub directly by each node, will not change the total number of LTEs during the upstream process. This only effects the number of wavelengths used. However, the number of wavelengths is not a factor of the network cost according to our assumptions. Thus, there are $n\left\lceil\frac{r}{g}\right\rceil$ unidirectional lightpaths established from each node to the hub in the upstream process and the total cost includes the LTEs used by the nodes to transmit traffic and the hub to receive traffic, which is $n\left\lceil\frac{r}{g}\right\rceil+n\left\lceil\frac{r}{g}\right\rceil=2 n\left\lceil\frac{r}{g}\right\rceil$.

Let us consider downstream now. The total amount of traffic units collected at the hub is $n r$. Since traffic bifurcation is allowed, the minimum number of wavelengths can be used to pack all the traffic, denoted by $\left\lceil\frac{n r}{g}\right\rceil$, which is also equal to the number of LTE ports required by the hub to transmit and by each node to receive. Each node employs a tap-andcontinue function which splits the optical signal and receives a small portion of power that is just enough to be detected and leave the rest of power to continue propagating on the ring. Such a power splitting function, performed by optical splitters, enables a broadcast service to be fulfilled by using only $n+1$ LTEs, one required at the hub and one required at each receiving node. Thus, broadcasting all of the traffic, which requires $\left\lceil\frac{n r}{g}\right\rceil$ wavelengths, will use a minimum cost of $(n+1)\left\lceil\frac{n r}{g}\right\rceil$ LTEs for the downstream traffic delivery.

To sum up, the resources consumed in both the upstream and downstream direction result in an overall minimum cost of $2 n\left\lceil\frac{r}{g}\right\rceil+(n+1)\left\lceil\frac{n r}{g}\right\rceil$ LTEs to achieve all-to-all communication.

\section{B. Application of Network Coding}

When using network coding, it is obvious that the hub is a perfect place to perform network coding, since all data has to be delivered to the hub first and then converted into electronic signals for grooming, and hence no additional LTEs will be needed for O-E-O conversion to perform network coding. Therefore, the encoding operation is performed at the hub and the decoding is done at each node. We can also consider this problem in the upstream and downstream contexts. Since upstream is unicast and no network coding is needed, the number of LTEs required in the upstream process remains the same. In order to save sub-wavelength channels in the downstream data delivery, we use the following coding scheme. Since each node needs to receive data from different $n-1$ nodes, then without implementing network coding but using splitters, each node has to receive all the data units from the hub, which is denoted by $n r$, in order to achieve minimum network cost. However, if a node receives linear combinations of the traffic instead of the original data, only $n-1$ linearly independent combinations are needed. By counting its own data in, each node has $n$ linearly independent combinations, 
from which original data of all other nodes can be decoded, given the coding coefficients are known. This is the basic idea for using network coding to save lightpaths and hence LTEs.

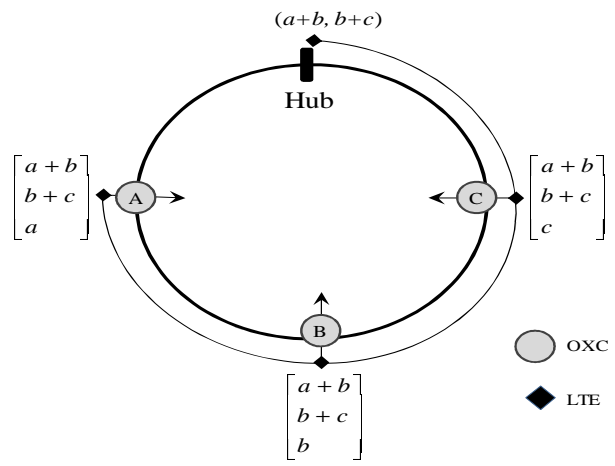

Fig. 2. Application of network coding in downstream in a single-hub ring

The example shown in Figure 2 illustrates how to use network coding in the downstream process. We suppose that the hub has received the traffic from nodes $A, B$ and $C$ whose data units are denoted by $a, b, c$, respectively. The grooming factor is 2 and transmission rate at each node is 1 . Hence, each wavelength is able to accommodate the traffic transmitted by two nodes. Instead of sending all the traffic $a, b$ and $c$ to each node on the ring, the hub encodes the data and generates code words $a+b$ and $b+c$ using modulo 2 addition and broadcast them. Hence, node $A$ will have combinations $a, a+b$ and $b+c$, where

$$
\left[\begin{array}{c}
a \\
a+b \\
b+c
\end{array}\right]=\left[\begin{array}{lll}
1 & 0 & 0 \\
1 & 1 & 0 \\
0 & 1 & 1
\end{array}\right]\left[\begin{array}{l}
a \\
b \\
c
\end{array}\right]
$$

Therefore, the coefficient matrix shown above has a full rank such that $a, b$ and $c$ can be decoded from the combinations. Following the same method, node $B$ and $C$ are also able to obtain all the original data $a, b, c$. Apparently, this coding scheme can be applied to the $n$-node case where the hub needs to generate $n-1$ linearly independent combinations which are also independent from all raw data units and broadcast them to each node.

Hence, in the case where the number of nodes is $n$ and the traffic rate associated with each node is $r$, the cost of upstream transmission does not change from the case without network coding, which is denoted by $2 n\left\lceil\frac{r}{g}\right\rceil$. In the downstream direction, however, the total traffic that the hub has to deliver is reduced to $(n-1) r$ which requires $\left\lceil\frac{(n-1) r}{g}\right\rceil$ wavelengths. The total cost of LTE ports in the downstream is $(n+1)\left\lceil\frac{(n-1) r}{g}\right\rceil$.

Therefore, the overall cost savings by the application of network coding in a single-hub ring with all-to-all traffic demand is $(n+1)\left(\left\lceil\frac{n r}{g}\right\rceil-\left\lceil\frac{(n-1) r}{g}\right\rceil\right) \geq 0$. The savings can be either in LTEs or network bandwidth, depending on the specific network scenarios as indicated above.

\section{Multiple Many-to-Many Groups}

In practice, the most common applications which use the all-to-all service mode are multimedia conferences and cooperative processing. Usually, there is more than one multimedia conference group simultaneously in the network. Thus, it is essential to consider multiple groups. In this scenario, the network nodes are divided into multiple groups, and within each group, nodes engage in all-to-all communication, while the traffic rates can be different in different groups.

1) Disjoint Groups: The definition of disjoint groups is straightforward - it refers to the case in which different groups do not share any common node. The problem of optimizing the network cost in a disjoint group is stated as follows: minimize the number of LTEs used in a single-hub unidirectional ring, given $m$ disjoint groups where each group $i(1 \leq i \leq m)$ has $n_{i}\left(n_{i} \geq 2\right)$ nodes and each node has to transmit $r_{i} \geq 1$ units of traffic to all the other nodes within the same group.

Let us consider the case without network coding first. The process is similar with the all-to-all case, which includes upstream and downstream process. The analysis still begins with the upstream process. Every group $i$ is independent from each other such that the total number of LTEs used is the sum of LTEs consumed by each group individually. Following the analysis in Section III.A, for each group $i$, the upstream consumes $2 n_{i}\left\lceil\frac{r_{i}}{g}\right\rceil$ LTEs, resulting in $\sum_{i=1}^{m} 2 n_{i}\left\lceil\frac{r_{i}}{g}\right\rceil$ in total.

In the downstream direction, the hub first grooms the traffic from the same group together. For group $i$, the number of wavelengths used to carry the traffic is $\left\lceil\frac{r_{i} n_{i}}{g}\right\rceil$, and $\left\lfloor\frac{r_{i} n_{i}}{g}\right\rfloor$ of them are filled up and sent back to the nodes belonging to the same group directly. Every lightpath used here is fully loaded and carries data for only one group. Thus, we only need to pay attention to the remaining portion of aggregate traffic for each group that cannot fill up a single wavelength if it exists, since it may need to be groomed with the traffic from other group(s) before delivery in order to save the lightpaths and hence LTEs. Each group has at most one piece of such traffic. This piece of traffic of group $i$ is equal to $n_{i} r_{i}-g\left\lfloor\frac{n_{i} r_{i}}{g}\right\rfloor$. We denote this portion of the traffic by $p_{i}$, where $0 \leq p_{i}<g$. Thus, the problem can be stated formally as follow:

Problem GMP: Given $m$ pieces of traffic, each of them with $p_{i}$ units and has to be received by the corresponding $n_{i}$ nodes, groom and multicast the traffic at the hub such that the total LTE ports used is minimized.

2) NP-completeness: In the optimal solution, any $p_{i}$ must not be bifurcated and packed into more than one wavelength. We can prove it by contradiction. Assume that one piece $p_{i}$ is split and assembled into two different wavelengths, which costs each node of group $i$ two LTE ports to receive. This results in $2 n_{i}$ LTE ports in total. If the hub uses a separate wavelength to send $p_{i}$, it only takes $n_{i}$ LTEs at the nodes and 1 more LTE at the hub. Since $2 n_{i}-\left(n_{i}+1\right)=n_{i}-1 \geq 0$, it means that any optimal solution can be transformed to be the solution without traffic bifurcation for the problem. Therefore, this problem turns into a special case of general traffic grooming problem in a ring network, which has been proven to be NP-complete by reduction from Bin Packing problem in polynomial time in [1].

3) Solutions without Network Coding: We now analyze the minimum network cost of many-to-many groups communication without employing network coding. The network cost of upstream transmission for $n$ groups has been derived in 2), which is $\sum_{i=1}^{m} 2 n_{i}\left\lceil\frac{r_{i}}{g}\right\rceil$. The cost of downstream transmission 
consists of two parts. The first part is the number of LTE ports used for broadcasting the lightpaths that are fully loaded for each individual group; the second part is the number of LTEs used for transmitting all the remaining traffic $p_{i}$. Since problem GMP is equivalent to the Bin Packing problem, which has been solved by many approaches in the literature, we consider two methods to obtain the minimum wavelengths in GMP. The first method is a heuristic algorithm based on First Fit Decreasing (FFD) [29]; In the second one, it is formulated by using Integer Linear Programming [30].

In the downstream transmission, for each group $i,\left\lfloor\frac{n_{i} r_{i}}{g}\right\rfloor$ wavelengths are fully utilized to pack the data, which takes $\left(n_{i}+1\right)\left\lfloor\frac{n_{i} r_{i}}{g}\right\rfloor$ LTEs. To sum up, the total number of LTEs used to transmit this portion of the traffic is $\sum_{i=1}^{m}\left(n_{i}+1\right)\left\lfloor\frac{n_{i} r_{i}}{g}\right\rfloor$. We use $W$ to denote the number of wavelengths used at the hub to accommodate the $p_{i}$ units of traffic of all the groups, which can be solved by either the heuristic or ILP described above. Hence, we need $W$ LTEs at the hub to transmit and 1 LTE for each node of group $i$ to receive $p_{i}$ if it exists, which is determined by a binary number, $\left\lceil\frac{n_{i} r_{i}}{g}\right\rceil-\left\lfloor\frac{n_{i} r_{i}}{g}\right\rfloor$. Therefore, the total cost of transmitting all the $p_{i}$ data units is equal to $W+\sum_{i=1}^{m}\left(\left\lceil\frac{n_{i} r_{i}}{g}\right\rceil-\left\lfloor\frac{n_{i} r_{i}}{g}\right\rfloor\right) \times n_{i}$.

Thus, the total network cost in both upstream and downstream for $m$ groups without network coding is:

$\sum_{i=1}^{m}\left(2 n_{i}\left\lceil\frac{r_{i}}{g}\right\rceil+\left(n_{i}+1\right)\left\lfloor\frac{n_{i} r_{i}}{g}\right\rfloor+\left(\left\lceil\frac{n_{i} r_{i}}{g}\right\rceil-\left\lfloor\frac{n_{i} r_{i}}{g}\right\rfloor\right) n_{i}\right)+W$.

4) Solutions with Network Coding: To apply network coding to single-hub ring networks, the hub acts as an encoder and generates $n_{i}-1$ code words for each group following the same coding scheme described in Fig. 2 with coefficients from $G F(2)$. The combinations of original data has the same rate as the original traffic, which is $r_{i}$. Thus, network coding can reduce the total traffic broadcast rate for each group $i$ by $r_{i}$. The upstream transmission consumes the same amount of resources as in the case without network coding, but the total traffic rate turns out to be $\left(n_{i}-1\right) r_{i}$ units for each group $i$ in the downstream process. Following the same computation rules used above, the network cost in this case also includes two parts. The first part is denoted by $\sum_{i=1}^{m}\left(n_{i}+1\right)\left\lfloor\frac{\left(n_{i}-1\right) r_{i}}{g}\right\rfloor$. In the second part, let $W^{\prime}$ denote the minimum number of wavelengths used at the hub to pack all the remaining traffic $p_{i}^{\prime}$ from each group after network coding.

Combining the cost spent in both upstream and downstream process gives us the total number of LTE ports with the application of network coding in a many-to-many traffic scenario, which is expressed as:

$$
\begin{gathered}
\sum_{i=1}^{m}\left(2 n_{i}\left\lceil\frac{r_{i}}{g}\right\rceil+\left(n_{i}+1\right)\left\lfloor\frac{\left(n_{i}-1\right) r_{i}}{g}\right\rfloor+\right. \\
\left.\left(\left\lceil\frac{\left(n_{i}-1\right) r_{i}}{g}\right\rceil-\left\lfloor\frac{\left(n_{i}-1\right) r_{i}}{g}\right\rfloor\right) \times n_{i}\right)+W^{\prime} .
\end{gathered}
$$

\section{Non-disjoint Groups}

Intuitively, non-disjoint groups denote that in a network, communication groups are not node disjoint and hence some nodes may belong to multiple groups. It is clear that in single-hub ring network, non-disjoint group scenario is the generalization of disjoint group case. Therefore, it follows that the traffic grooming problem with non-disjoint groups is also $N P$-complete by reduction to the special disjoint group case.

Since the network cost is exactly the same for both cases with and without network coding in the upstream direction, we will just focus on the cost analysis of the downstream process. Assume after the hub grooms the data sent from the same group, there may be a remaining traffic $p_{i}$ for each group $i$ that can not be able to fill up a wavelength at the hub and the hub will groom them together. The difference between this case and the node-disjoint group case is that by grooming the $p_{i}$ of the groups that share some common nodes together, it saves some LTEs at the common receiving nodes of these groups. Therefore, the hub may have preference to groom the $p_{i}$ of the groups that have more common nodes together.

In order to achieve the optimal solution of the total number of LTE required in downstream direction, we formulate the problem by using integer linear programming. Given the network topology, traffic rate and number of nodes of each group, as well as the number of nodes shared by any pair of groups, we could achieve the optimal solution for both cases with and without using network coding. The only difference between the two cases is the remaining traffic $p_{i}$ of each group $i$.

Without employing network coding in the network, for each group $i$, the total number of sub-wavelength circuits needed to broadcast at the hub is denoted by: $t r_{i}=\sum_{k=1}^{N} m_{k}^{i} r_{i}, \quad \forall i \leq$ $M$, where $N$ is the total number of nodes, $M$ denotes the total number of groups and binary $m_{k}^{i}$ is equal to 1 if node $k$ is a member of group $i$.

Whereas in the case where network coding is applied, the total number of circuits for each group $i$ collected at the hub is equal to $t r_{i}=\left(\sum_{k=1}^{N} m_{k}^{i}-1\right) r_{i}, \forall i \leq M$.

Accordingly, for both cases, the remaining portion of traffic flow for group $i$ is denoted by:

$$
p_{i}=t r_{i}-g\left\lfloor\frac{t r_{i}}{g}\right\rfloor,\left(0 \leq p_{i}<g\right) .
$$

Therefore, the total number of LTEs required at the hub and nodes for remaining traffic communication can be obtained by solving the following ILP:

\begin{tabular}{ll}
\hline \hline Symbol & Meaning \\
\hline$W$ & $\begin{array}{l}\text { the maximum number of the wavelengths used in the } \\
\text { formulation } \\
\text { the remaining circuit } p_{i} \text { from group } i \text { that is allocated into } \\
p_{i}^{w}\end{array}$ \\
$h^{w}$ & $\begin{array}{l}\text { wavelength } w \text { at the hub } \\
\text { a binary variable that equals } 1 \text { if a LTE is used at the hub } \\
s_{i, j}^{w}\end{array}$ \\
$n s_{i, j}$ & $\begin{array}{l}\text { a binary variable that equals } 1 \text { if wavelength } w \text { carries } \\
\text { circuits for both groups } i \text { and } j \\
\text { the number of nodes shared between group } i \text { and } j, \text { a } \\
\text { constant given in the problem }\end{array}$ \\
\end{tabular}

TABLE I

VARIABLES AND PARAMETERS IN THE ILP FORMULATION FOR DOWNSTREAM PROCESS IN SINGLE-HUB RING 
- Objective:

Minimize $\sum_{w=1}^{W} h^{w}+\sum_{i=1}^{M} \sum_{w=1}^{W} p_{i}^{w} n_{i}-\sum_{w=1}^{W} \sum_{i=1}^{M} \sum_{j>i}^{M} s_{i, j}^{w} n s_{i, j}$

- Subject to:

$$
\begin{array}{r}
h^{w} \geq \frac{1}{M} \sum_{i=1}^{M} p_{j}^{w}, \quad \forall w ; \\
\sum_{i=1}^{M} p_{i}^{w} \times p_{i} \leq g, \quad \forall w ; \\
\sum_{w=1}^{W} p_{i}^{w}=1, \quad \forall i ; \\
p_{i}^{w}+p_{j}^{w}-1 \leq s_{i, j}^{w} \leq \frac{1}{2}\left(p_{i}^{w}+p_{j}^{w}\right), \\
\forall i<j \leq M, w \leq W .
\end{array}
$$

The objective function consists of three terms. The first term denotes the total number of LTEs required at the hub. the second term sums up the number of LTEs required for all the nodes of group $i$ to receive $p_{i}$ without considering node sharing. It means that we may count a LTE twice if a node $k$ belongs to both group $i$ and $j$ and $p_{i}$ and $p_{j}$ happen to be groomed in the same wavelength. Therefore, in the third term, we subtract the portion of the cost counted redundantly.

Constraint 1 ensures that if wavelength $w$ is used to accommodate traffic of any group, the corresponding lightpath should be set up at the hub. The wavelength capacity constraint is satisfied by equation 2 . Constraint 3 makes sure that each remaining traffic $p_{i}$ should be allocated into a wavelength. Constraint 4 ensures that if the circuits of group $i$ and $j$ are groomed into the same wavelength $w$, then $s_{i, j}^{w}$ should be 1 .

Therefore, adding up the LTEs cost obtained from ILP to the network cost of provisioning the rest of the traffic will yield the total cost in this downstream process, represented by

$$
\sum_{i=1}^{M}\left(n_{i}+1\right)\left\lfloor\frac{t r_{j}}{g}\right\rfloor+L T E_{I L P} .
$$

where $L T E_{I L P}$ denotes the number of LTEs obtained by solving the ILP for the remaining traffic communication.

\section{Cost Analysis in Un-Hubbed Unidirectional RINGS}

Following the same sequence of the previous section, we will first investigate the traffic grooming problem in an unhubbed ring with all-to-all traffic and then generalize it to many-to-many group communication. All the assumptions made in the single-hub ring case remain except that a hub is not used.

\section{A. Uniform all-to-all Traffic}

The problem can be defined as follows: given a grooming factor $g$ and a group of $n$ nodes, each of which has $r$ units of traffic, find the minimum number of LTEs required to fulfill all-to-all communication.
First, the lower bound and upper bound of the special case of unitary traffic, i.e. $r=1$, can be derived if optical splitters are allowed. Each node needs at least $\left\lceil\frac{n-1}{g}\right\rceil$ LTEs to receive and one LTE to send traffic. Thus, the lower bound of network cost is $\left(1+\left\lceil\frac{n-1}{g}\right\rceil\right) n$. However, if no traffic is groomed, the maximum number of LTEs requested is equal to $n^{2}$, which can be considered as the upper bound on the cost of this special case.

For the general case of arbitrary $r$, we propose a multihub approach to serve all-to-all demands while minimizing the total number of LTEs. The approach can be done in two steps:

1) Divide the nodes into a number of sub-groups such that the aggregated traffic of each sub-group is just enough to fill up a wavelength;

2) Choose one node of each sub-group as a hub to groom the traffic from other group members and broadcast the groomed traffic to all the nodes on the ring.

Unlike the situation of a single-hub ring where all nodes send traffic to the same hub, in an un-hubbed ring, once enough traffic is groomed to fill up a wavelength at a node, then this node will set up a lightpath and broadcast the data to the other $n-1$ nodes on the ring. This node is called a "hub" and we may have multiple hubs on the ring. The number of such hubs is equal to the number of wavelengths to accommodate all the traffic. The minimum number of wavelengths that can be achieved equals $\left\lceil\frac{n r}{g}\right\rceil$. In addition, we only consider the case where $r<g$, since if $r \geq g$, the excess traffic $(r-r \bmod g)$ of each node can fill up separate $\left\lfloor\frac{r}{g}\right\rfloor$ wavelengths without traffic grooming. In this case, we only need to consider the remaining traffic, denoted by $r-g\left\lfloor\frac{r}{g}\right\rfloor$, which is less than $g$. Thus, there is no need to consider the case where $r>g$.

We analyze the network cost in two different cases: when $g$ is a multiple of $r$ or not. In fact, the case when $g$ is a multiple of $r$ is a special case of the other case in terms of the solution. Thus we will only discuss the case when $g$ is not a multiple of $r$ in detail.

In the case when $g$ is not a multiple of $r$, a wavelength cannot be filled up without traffic bifurcation. Minimizing the total number of wavelengths used for broadcasting with traffic bifurcation will result in the minimum number of broadcast cycles. However, each traffic split introduces two additional LTEs, because when a piece of traffic is allocated into two wavelengths instead of one, this requires two lightpaths to carry and results in two additional LTEs (one transmitter and one receiver). Alternatively, if we do not split any traffic, we cannot guarantee that the number of broadcast cycles is at a minimum. Each broadcast uses $n$ LTEs, which means that saving one wavelength will save $n$ LTEs. Therefore, there is a trade-off between the number of traffic splits and the total number of broadcast cycles. In the multi-hub approach, the minimum network cost is obtained by taking the minimum value of the solutions obtained from the two scenarios where we split and we do not split the traffic.

First, we consider the case without traffic bifurcation. Each wavelength can accommodate traffic from at most $\left\lfloor\frac{g}{r}\right\rfloor$ nodes, 
denoted by $k$. A small amount of bandwidth equal to, $g-$ $k r$, is wasted on each wavelength. Then, the total number of broadcast cycles is $\left\lceil\frac{n}{k}\right\rceil$. Among them, each of $\left\lfloor\frac{n}{k}\right\rfloor$ broadcast cycles fully utilizes a wavelength with a small wastage of bandwidth, and each of such cycles requires $2(k-1)+n$ LTEs for transmitting and receiving, which results in a total $\left\lfloor\frac{n}{k}\right\rfloor(2(k-1)+n)$ LTEs for the first $\left\lfloor\frac{n}{k}\right\rfloor$ broadcast cycles. However, the number of nodes remaining in the last cycle is $\left(\left\lceil\frac{n}{k}\right\rceil-\left\lfloor\frac{n}{k}\right\rfloor\right)\left(n-k\left\lfloor\frac{n}{k}\right\rfloor\right)$ where $\left(\left\lceil\frac{n}{k}\right\rceil-\left\lfloor\frac{n}{k}\right\rfloor\right)$ is a binary number to indicate if there are some nodes left in the last cycle whose number is less than $k$ and their aggregated traffic cannot fill up one wavelength. Hence, the number of LTEs needed in the last broadcast cycle is $\left(\left\lceil\frac{n}{k}\right\rceil-\left\lfloor\frac{n}{k}\right\rfloor\right)\left(2\left(n-k\left\lfloor\frac{n}{k}\right\rfloor-1\right)+n\right)$.

Therefore, the number of LTEs in this scenario is given by combining the cost in all broadcast cycles, namely:

$$
\left\lceil\frac{n}{k}\right\rceil\left(3 n-2 k\left\lfloor\frac{n}{k}\right\rfloor-2\right)+\left\lfloor\frac{n}{k}\right\rfloor\left(2 k\left\lfloor\frac{n}{k}\right\rfloor-2 n+2 k\right),
$$

where $k=\left\lfloor\frac{g}{r}\right\rfloor$. This general solution can also be applied to the case when $g$ is a multiple of $r$.

In the second scenario where traffic bifurcation is applied, the minimum number of wavelengths to accommodate all the traffic can be achieved, which is given by $\left\lceil\frac{n r}{g}\right\rceil$, which also equals the minimum number of broadcast cycle. Let $\left\lceil\frac{n r}{g}\right\rceil=w_{\min }$. Hence, the number of LTEs employed for a broadcast can by obtained by $n w_{\min }$. However, we know that traffic splitting was used to achieve this due to the assumption that $g$ is not a multiple of $r$. Since each traffic split uses two additional LTEs, the problem of minimizing the total number of LTEs actually turns out to be a problem of minimizing the number of traffic splits in order to groom the traffic on the minimum number of wavelengths, $w_{\min }$. This problem has been solved by an iterative algorithm proposed in [1]. In each iteration, three steps are processed:

1) Fill each of $w_{\min }$ wavelengths with $\left\lfloor\frac{g}{r}\right\rfloor r$ loads. Therefore, the unused capacity left on each wavelength becomes $g^{\prime}=g-\left\lfloor\frac{g}{r}\right\rfloor r$ and the number of nodes whose traffic has not been assigned is equal to $n^{\prime}=n-$ $\left\lfloor\frac{g}{r}\right\rfloor w_{\text {min }}$

2) We have $n^{\prime}<w_{\text {min }}$ and $r>g^{\prime}$ from the first step. And then allocate $g^{\prime}$ units of traffic of each unassigned node to $n^{\prime}$ wavelengths, respectively, to fill them up.

3) As a result, only $w_{\min }-n^{\prime}$ wavelengths still have $g^{\prime}$ units of capacity available. Update $w_{\min }:=w_{\min }-n^{\prime}$, $r:=r-g^{\prime}$ and $n=n^{\prime}$, and then repeat the three steps until all the traffic is assigned.

We use the algorithm here to obtain the minimum number of traffic splits in this situation, denoted by $s p_{\min }$, given the minimum number of wavelengths used. The number of broadcast cycles determines the number of hubs in the ring, which is also equal to $w_{\text {min }}$. If no traffic split happens, collecting traffic at those hub nodes from other nodes before broadcast requires $2\left(n-w_{\text {min }}\right)$ LTEs. However, each traffic split increases the number of LTEs by 2. Thus, the total number of LTEs in this collection process is $2\left(n-w_{\text {min }}+s p_{\text {min }}\right)$. In addition to the LTEs used for broadcasting, denoted by $n w_{m i n}$, the total number of LTEs used in this scenario with traffic bifurcation is $2\left(n-w_{\text {min }}+s p_{\text {min }}\right)+n w_{\min }$.
Therefore, taking the minimum of the two solutions obtained in the two scenarios above will give us the overall minimum network cost. Thus, the number of LTEs of all-to-all traffic without network coding is:

$$
\begin{aligned}
\min \{ & \left\lceil\frac{n}{k}\right\rceil\left(3 n-2 k\left\lfloor\frac{n}{k}\right\rfloor-2\right)+\left\lfloor\frac{n}{k}\right\rfloor\left(2 k\left\lfloor\frac{n}{k}\right\rfloor-2 n\right. \\
& \left.+2 k), \quad 2\left(n-w_{\text {min }}+s p_{\min }\right)+n w_{\min }\right\}
\end{aligned}
$$

where $k=\left\lfloor\frac{g}{r}\right\rfloor, w_{\min }=\left\lceil\frac{n r}{g}\right\rceil$, and $s p_{\min }$ is the minimum number of traffic splits obtained by the iterative algorithm proposed in [1], given $w_{\min }$.

\section{B. Application of Network Coding}

In order to save network cost by performing network coding, a node should be chosen to collect all the original data. Thus, we propose a one-hub scheme in which only one node acts as a hub. The traffic is gathered and encoded at this node following the same coding scheme proposed in Section III.B, where the network context is a single-hub ring. The hub can be selected from any node in the ring.

Hence, in the upstream direction, every node sending traffic to the hub consumes $2(n-1)$ LTEs. $n-1$ linearly independent code words with traffic rate $r$ are generated and packed into $\lceil r(n-1) / g\rceil$ wavelengths. In the downstream direction, the minimum number of broadcast cycles can be achieved, which also equals to $\lceil r(n-1) / g\rceil$. Each broadcast costs $n$ LTEs such that the transmission in the downstream direction takes total $n\lceil r(n-1) / g\rceil)$ LTEs.

Therefore, the total network cost with network coding using one-hub scheme is: $2(n-1)+n\lceil r(n-1) / g\rceil$.

Though one-hub scheme can save LTEs in downstream direction, it uses a few more LTEs in the upstream process compared to the multi-hub approach. Thus, the total number of LTEs consumed in both upstream and downstream directions may not always saved by employing one-hub scheme, depending on the specific network scenario. However, given the traffic demands, we can always use the multi-hub approach to solve the problem without applying network coding. Therefore, by comparing the solutions yielded by the one-hub and multi-hub approaches, we choose the solution of the minimum value.

Thus, assuming that $L T E_{\text {multi }}$ denote the solution obtained from the multi-hub approach, the total network cost in unhubbed rings with $n$ nodes and all-to-all traffic demands $r$ while applying network coding is:

$$
\min \left\{2(n-1)+n\lceil r(n-1) / g\rceil, \quad L T E_{m u l t i}\right\} .
$$

\section{Multiple Many-to-Many Groups}

We now extend the all-to-all communication to multiple many-to-many disjoint groups on an un-hubbed unidirectional ring. Since no node is shared by more than one group, there is no common hub being able to groom the traffic from different groups together. Thus, each group can be provisioned independently, and the total network cost is the sum of the cost of all groups.

Suppose there are $m$ groups in an un-hubbed ring and each group $i$ has $n_{i}$ nodes with each node in the group sourcing $r_{i}$ traffic units, for $1 \leq i \leq m$. The minimum network cost in 
terms of LTEs can be represented based on whether network coding is employed or not:

In the case where no network coding is employed, the total network cost in terms of the number of LTEs is:

$$
\begin{gathered}
L T E_{\text {multi }}^{i}=\sum_{i=1}^{m} \min \left\{\left\lceil\frac{n_{i}}{k_{i}}\right\rceil\left(3 n_{i}-2 k_{i}\left\lfloor\frac{n_{i}}{k_{i}}\right\rfloor-2\right)+\left\lfloor\frac{n_{i}}{k_{i}}\right\rfloor *\right. \\
\left.\left(2 k_{i}\left\lfloor\frac{n_{i}}{k_{i}}\right\rfloor-2 n_{i}+2 k_{i}\right), 2\left(n_{i}-w_{\text {min }}^{i}+s p_{\text {min }}^{i}\right)+n_{i} w_{\text {min }}^{i}\right\},
\end{gathered}
$$

where $k_{i}=\left\lfloor\frac{g}{r_{i}}\right\rfloor, w_{\text {min }}^{i}=\left\lceil\frac{n_{i} r_{i}}{g}\right\rceil$, and $s p_{\text {min }}^{i}$ is the minimum number of traffic splits of group $i$ given $w_{\text {min }}^{i}$.

In the case of using network coding, the total network cost in terms of the number of LTEs is:

$$
\sum_{i=1}^{m} \min \left\{2\left(n_{i}-1\right)+n_{i}\left\lceil r_{i}\left(n_{i}-1\right) / g\right\rceil, \quad L T E_{\text {multi }}^{i}\right\},
$$

\section{Non-disjoint Groups}

We also consider group non-disjointness in an un-hubbed ring network where multiple groups may overlap. Two network scenarios, either employing network coding or not, will be discussed, respectively. We will first consider the case of applying network coding.

1) With Network Coding: In order to perform network coding on the traffic for a group, one member node should be chosen to play the role of the hub as in hubbed-rings. The encoding operation remains the same as in the disjoint groups case and the total number of circuit needed to broadcast for each group $i$ is: $t r_{i}=\left(\sum_{k} m_{k}^{i}-1\right) r_{i}$, where $m_{k}^{i}$ is equal to 1 if node $k$ is a member of group $i$. The traffic requires $\left\lceil\frac{t r_{i}}{g}\right\rceil$ wavelengths to accommodate group $i$. Among them, $\left\lfloor\frac{t r_{i}}{g}\right\rfloor$ are filled up and the corresponding number of LTEs used to broadcast this portion of traffic for group $i$ is $n_{i}\left\lfloor\frac{t r_{i}}{g}\right\rfloor$.

Let $p_{i}=t r_{i}-\left\lfloor\frac{t r_{i}}{g}\right\rfloor$ denote the remaining piece of traffic of group $i$, and each hub needs to broadcast $p_{i}$ to the nodes within the same group. Since some nodes are shared between distinct groups, if one node can act as the hub for mutilple groups and the $p_{i}$ of those groups happen to be packed into the same wavelength, LTE cost is reduced by sharing this hub. Notice that if two groups cannot share a hub node, though if their $p_{i}$ are small enough to be allocated into one wavelength, we should not do it. The reason is as follow: given two groups, $i$ and $j$, a node of group $i$ needs one LTE to send the $p_{i}$ to the hub node of group $j$ in order to groom the traffic together, which results in another LTE at the receiving node. Thus, such grooming action results in two more LTEs for an extra delivery. In order to reduce the cost, we do not perform the traffic grooming in such case where two groups have no common node even if their remaining traffic can be packed into the same wavelength.

Since the cost of establishing the fully loaded lightpaths is fixed, we only need to concentrate on the cost of provisioning $p_{i}$ of each group $i$. In order to minimize the number of LTEs used to broadcast all $p_{i}$, we propose a heuristic algorithm, $H u b$ Sharing Minimization (HSM), to allocate all the $p_{i}$ based on the situation where the nodes are assigned to different groups, from which we can calculate how many LTEs can be saved by sharing the hub nodes and receiving nodes, and consequently the total number of LTEs required. Prior to the description of $H S M$, we introduce some symbols used in the algorithm and the preliminary processes.

1) First, we create a bipartite graph $G=(V, E)$, where $V=X \bigcup Y$ and $X \bigcap Y=\emptyset$. Partition $X$ represents the groups and partition $Y$ represents the nodes. An undirected link $e \in E$ is established between $i \in X$ and $j \in Y$ if node $j$ is a member of group $i$ in the ring;

2) Each vertex $i \in X$ is assigned a weight value equal to $p_{i}$. The degree of vertex $i$ is denoted by $d_{i}$ and its neighbors, defined as the vertices in $Y$ that connect to it, are denoted by $N_{i}$;

3) All the vertices in $X$ will be gradually divided into multiple sets. Each set, denoted by $S_{k},(1 \leq k \leq\|X\|)$, is composed of a number of vertices that must have a common neighbor in $Y$, say $j$, and their corresponding traffic $p_{i}$ are assigned in one wavelength together. We use $S N\left(S_{k}\right)$ to denote the total number of times that the vertices in $S_{k}$ share common neighbors. Let us take an example as shown in Fig.3. If the first set of $X$ is $S_{1}=\{1,2,3\}$, and then $S N\left(S_{1}\right)=2+1=3$, since vertex 1,2 and 3 share a common neighbor $B$ in $Y$ and vertex 2 and 3 also share node $C$ in $Y$. We say that vertex $B$ is shared twice while vertex $C$ is shared once. Thus, the total number of sharing is equal to 3 .

We assume that the ring network has been transformed to a bipartite graph $G=(V, E)$ described in 1) and the procedure of algorithm HSM is shown in Algorithm 1:

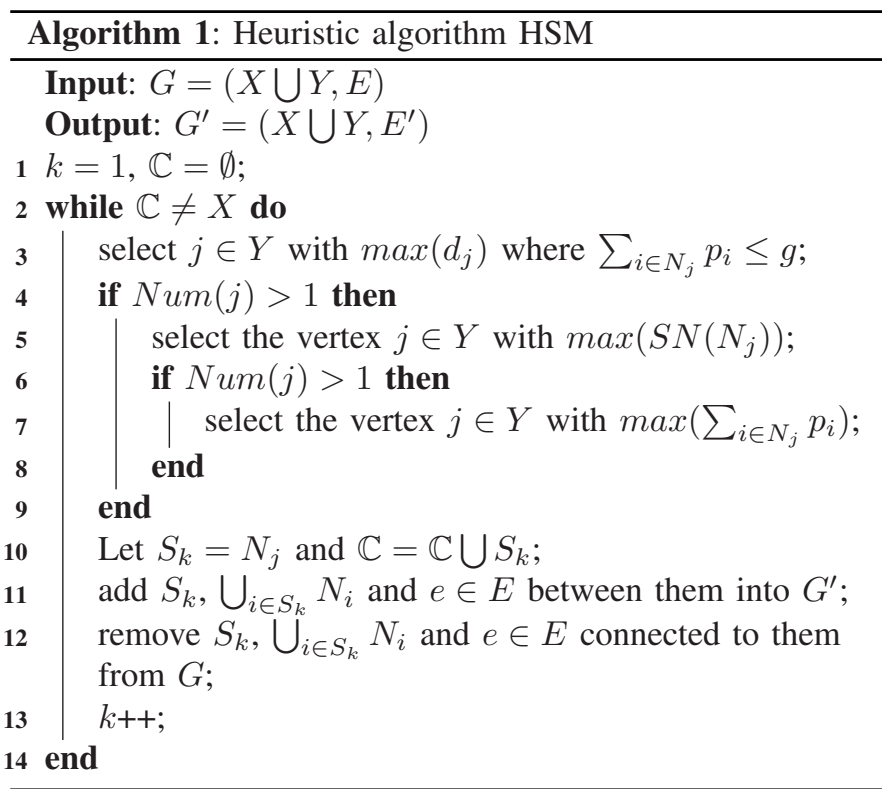

Basically, heuristic HSM is a greedy based algorithm and the idea behind it is to divide all the groups into several sets, and each set represents a combination of group(s) that groom their traffic $p_{i}$ into the same wavelength and share the same hub node. For each group set $S_{i}$, the algorithm tries to choose the groups that share the most number of nodes such that the greatest number LTEs can be saved at both hubs and receivers. which is equal to $S N\left(S_{i}\right)$.

The example shown in Figure 3 illustrates how HSM works 
and how LTEs can be saved. Figure 3 shows the bipartite graph $G$ mapping from a ring topology, in which we assume there are five groups represented by the vertices $1,2,3,4$ and 5 in partition $X$ and each $p_{i}$ is indicated by the value above the vertex. The vertices in partition $Y$ represent the network nodes. Both solid and dash edges show which node belongs to which group in the ring. In the first iteration, vertex $B \in Y$ is picked with the largest degree, since this node is shared by the most number of groups. Since $p_{1}+p_{2}+p_{3}=7<g$, groups 1,2 and 3 , defined as set 1 , groom their traffic together in one wavelength at the common hub node $B$ and the total number of LTEs saved by selecting this set is equal to $S N\left(S_{1}\right)$, which is 4 . Then the set 1 is separated from $G$ by removing two dash lines, $(3, G)$ and $(4, D)$. In the second step, vertex $F$ is picked and the vertices 4 and 5 are grouped as set 2 . Since node $F$ is the only common node for them, only one LTE is saved at this node. Thus, the bipartite graph $G$ is divided into two group sets and the total number of saved LTEs is equal to four.

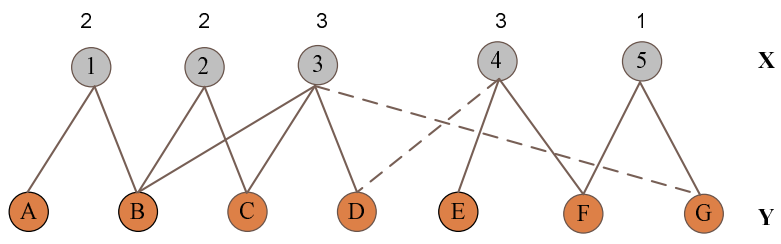

Fig. 3. An example of the procedure of $H S M$ in which $g=8$

We now derive the time complexity of this algorithm. We assume that the number of groups is denoted by $|X|$ whereas the number of nodes is denoted by $|Y|$ and each group has a constant number of members and each node is shared by a constant number of groups. Hence, each $N_{i}$ and $d_{i}$ is constant number. Thus, the complexity of the algorithm is dominated by the computation in line 3 , since line 4-13 take a constant computation time, denoted by $c$. The worst scenario is when no group shares any common node and the computation cost of line 3 is $|Y|$ and the total number of iterations in the while loop is $|X|$. Therefore, the complexity of algorithm HSM is $O(|X| *(|Y|+c))=O(|X| *|Y|)$.

If we denote total number of saved LTEs obtained from $H S M$ by $h_{s}$, we can then derive the total number of LTEs in both upstream and downstream processes:

In the upstream process, $\sum_{i} 2\left(n_{i}-1\right)$ LTEs are required to collect the data at the hub of each group.

In the downstream process, without considering group sharing, each group $i$ uses $n_{i}\left\lceil\frac{t r_{i}}{g}\right\rceil$ to broadcast the encoded data, which costs $\sum_{i} n_{i}\left\lceil\frac{t r_{i}}{g}\right\rceil$ LTEs in total. However, sharing member nodes among groups will save $h_{s}$ LTEs given by the heuristic HSM.

Therefore, the total number LTEs used in this scenario is given by:

$\sum_{i} 2\left(n_{i}-1\right)+n_{i}\left\lceil\frac{t r_{i}}{g}\right\rceil-h_{s}$, where $t r_{i}=\left(\sum_{k} m_{k}^{i}-1\right) r_{i}$.

2) Without Network Coding: In the scenario where no coding is employed, we can use the same approach employed to derive the cost for node disjoint case without application of network coding, described in Section.IV.C, to obtain the cost for this scenario. The only difference between two cases is that we can further reduce the cost of LTEs in this scenario by sharing the common hubs among distinct groups if they have common nodes by using the algorithm HSM to allocate all the remaining traffic $p_{i}$ for all the groups, where $p_{i}$ is derived as follows:

For any group $i$

- In the case where no traffic bifurcation is applied, $p_{i}=$ $r_{i}\left(n_{i}-k_{i}\left\lfloor\frac{n_{i}}{k_{i}}\right\rfloor\right)$, where $k_{i}=\left\lfloor\frac{g}{r_{i}}\right\rfloor$.

- In the case where traffic bifurcation is applied, $p_{i}=t r_{i}$ $\left\lfloor\frac{t r_{i}}{g}\right\rfloor$, where $t r_{i}=\sum_{k} m_{k}^{i} r_{i}$.

Thus, we assume the number of LTEs saved by sharing the common hubs among different groups is denoted by $h_{s}$ and $h_{s}^{\prime}$, solved by $H S M$, for the cases without and with traffic bifurcation, respectively. By subtracting $h_{s}$ and $h_{s}^{\prime}$ from the costs in node disjoint group case for both scenarios and taking the minimum value, we obtain the number of LTEs required for the many-to-many communication in this nondisjoint group case:

$$
\begin{array}{r}
\min \left\{\sum _ { i = 1 } ^ { m } \left(\left\lceil\frac{n_{i}}{k_{i}}\right\rceil\left(3 n_{i}-2 k_{i}\left\lfloor\frac{n_{i}}{k_{i}}\right\rfloor-2\right)+\right.\right. \\
\left.\left\lfloor\frac{n_{i}}{k_{i}}\right\rfloor\left(2 k_{i}\left\lfloor\frac{n_{i}}{k_{i}}\right\rfloor-2 n_{i}+2 k_{i}\right)\right)-h_{s}, \\
\left.\sum_{i=1}^{m}\left(2\left(n_{i}-w_{\text {min }}^{i}+s p_{\text {min }}^{i}\right)+n_{i} w_{\text {min }}^{i}\right)-h_{s}^{\prime}\right\}
\end{array}
$$

where $k_{i}=\left\lfloor\frac{g}{r_{i}}\right\rfloor, w_{\text {min }}^{i}=\left\lceil\frac{n_{i} r_{i}}{g}\right\rceil, s p_{\text {min }}^{i}$ is the minimum number of traffic splits of group $i$ given $w_{\text {min }}^{i}$.

\section{NUMERICAL RESUlTS}

Based on the theoretical solutions obtained above, we compare the results of two different cases - with or without network coding - in various network scenarios and under different traffic conditions. Since all-to-all communication is a special case of multiple many-to-many group cases where the number of groups is equal to 1 , in this section we consider more general cases in which $m \geq 1$.

Each network scenario is generated according to the following assumptions:

1) The number of many-to-many groups in the ring network is fixed at $m=10$;

2) The number of nodes per group is lower bounded by 2 and upper bounded by a given positive integer value $n_{\max }$ and is uniformly distributed in $\left[2, n_{\max }\right]$;

3) The traffic rate of each node is lower bounded by 1 and upper bounded by a given positive integer value $r_{\max }$ and is uniformly distributed in $\left[1, r_{\max }\right]$;

\section{A. Single-hub Ring}

Since network cost in the upstream direction in single-hub networks is always the same whether or not network coding is used, we only compare the cost factors in the downstream direction.

Table II shows the heuristic and exact solutions of the number of LTE ports in different network scenarios. Three 
TABLE II

THE COMPARISON OF DOWNSTREAM NETWORK COST OF DISJOINT GROUPS IN THE SINGLE-HUB RING WITH G=4,8 AND 16

\begin{tabular}{|c|c|c|c|c|c|c|c|c|c|c|c|c|c|}
\hline & & (Traffic,nodes) & $(2,20)$ & $(2.5,20)$ & $(2,30)$ & $(2.5,30)$ & $(2,40)$ & $(2.5,40)$ & $(2,50)$ & $(2.5,50)$ & $(2,60)$ & $(2.5,60)$ & $(2,70)$ \\
\hline \multirow{4}{*}{$g=4$} & \multirow[t]{2}{*}{ FFD } & Without NC & 36.8 & 42.6 & 71.2 & 84.8 & 122.4 & 146.0 & 184.2 & 220.4 & 258.0 & 311.9 & 343.1 \\
\hline & & With NC & 25.5 & 26.7 & 57.5 & 64.5 & 98.3 & 115.4 & 158.3 & 186.3 & 223.6 & 268.9 & 307.5 \\
\hline & \multirow[t]{2}{*}{ ILP } & Without NC & 36.8 & 42.6 & 71.2 & 84.8 & 122.4 & 146.0 & 184.2 & 220.4 & 258.0 & 311.9 & 343.1 \\
\hline & & With NC & 25.5 & 26.7 & 57.5 & 64.5 & 98.3 & 115.4 & 158.3 & 186.3 & 223.6 & 268.9 & 307.5 \\
\hline \multirow{4}{*}{$\mathrm{g}=8$} & \multirow[t]{2}{*}{ FFD } & Without NC & 25.5 & 26.7 & 46.1 & 51.4 & 73.2 & 84.0 & 101.0 & 119.6 & 144.3 & 170.6 & 191.1 \\
\hline & & With NC & 23.0 & 23.6 & 40.3 & 43.2 & 61.5 & 70.0 & 91.8 & 105.9 & 126.9 & 150.0 & 172.5 \\
\hline & \multirow[t]{2}{*}{ ILP } & Without NC & 25.5 & 26.7 & 46.1 & 51.4 & 73.2 & 84.0 & 101.0 & 119.6 & 144.3 & 170.6 & 191.1 \\
\hline & & With NC & 23.0 & 23.6 & 40.3 & 43.2 & 61.4 & 70.0 & 91.8 & 105.9 & 126.8 & 150.0 & 172.5 \\
\hline \multirow{4}{*}{$g=16$} & \multirow[t]{2}{*}{ FFD } & Without NC & 23.0 & 23.5 & 34.3 & 35.4 & 49.2 & 54.8 & 66.7 & 75.2 & 88.3 & 102.8 & 115.8 \\
\hline & & With NC & 21.9 & 22.0 & 33.0 & 33.7 & 44.1 & 47.8 & 55.9 & 69.9 & 81.2 & 93.6 & 106.0 \\
\hline & \multirow[t]{2}{*}{ ILP } & Without NC & 23.0 & 23.5 & 34.3 & 35.4 & 49.2 & 54.7 & 66.6 & 75.2 & 88.3 & 102.8 & 115.8 \\
\hline & & With NC & 21.9 & 22.0 & 33.0 & 33.7 & 44.0 & 47.8 & 55.8 & 69.9 & 81.2 & 93.6 & 106.0 \\
\hline
\end{tabular}

TABLE III

THE COMPARISON OF DOWNSTREAM NETWORK COST OF NON-DISJOINT GROUPS IN THE SINGLE-HUB RING WITH G=8 AND 16

\begin{tabular}{|c|c|cccccccccccc|}
\hline & (Traffic,nodes) & $(2,20)$ & $(3,20)$ & $(2,30)$ & $(3,30)$ & $(2,40)$ & $(3,40)$ & $(2,50)$ & $(3,50)$ & $(2,60)$ & $(3,60)$ & $(2,70)$ & $(3,70)$ \\
\hline $\mathrm{g}=8$ & Without NC & 20.2 & 27.9 & 39.5 & 52.6 & 64.9 & 102.4 & 91.0 & 147.5 & 128.3 & 197.9 & 177.5 & 212.0 \\
& With NC & 14.8 & 23.4 & 34.4 & 45.4 & 56.2 & 85.2 & 82.3 & 115.2 & 134.2 & 176.0 & 163.4 & 190.1 \\
\hline $\mathrm{g}=16$ & Without NC & 15.2 & 18.1 & 30.3 & 34.1 & 39.8 & 57.7 & 56.1 & 77.3 & 75.3 & 117.7 & 113.1 & 135.8 \\
& With NC & 11.0 & 12.2 & 25.5 & 28.7 & 33.3 & 48.9 & 50.7 & 68.0 & 69.3 & 104.6 & 103.1 & 125.9 \\
\hline
\end{tabular}

different grooming factors, $g$, are used. Each network scenario is represented by a pair of numbers in the parenthesis. The first number denotes the average traffic rate transmitted at each node, derived from $\left(1+r_{\max }\right) / 2$ and the second number denotes the average total number of nodes in the ring network, derived from $\left(2+n_{\max }\right) m / 2$ where $m=10$ based on the properties of uniform distribution. For instance, given a scenario where $r_{\max }=3$ and $n_{\max }=4$, this maps to the case where the average traffic rate and average total nodes are represented by $(2,30)$ in the table. In each scenario, upper bounds $r_{\max }$ and $n_{\max }$ are fixed, but the actual $n_{i}$ and $r_{i}$ for each group $i \leq m$ are randomly chosen between the lower bounds and upper bounds for each single experiment. Each network cost value is obtained by taking the average result of 500 independent experiments associated with a unique network scenario.

Under the same network scenarios and grooming factors, the table shows that the exact network cost is almost the same as its heuristic counterpart in most cases. There are only a few cases that the heuristic solutions are a little bit greater than the optimum due to the small difference between the exact and heuristic solutions of the problem GMP.

We can observe that the network cost increases in proportion of the traffic rates and the number of nodes. Network coding can save network cost in all cases where the grooming factor $g=4,8$ and 16. The relative savings of the network cost, denoted by the ratio of cost savings to the network cost without applying network coding, are almost the same under different network traffic conditions with the same $g$. However, the incremental saving of the network cost decreases as $g$ increases. The overall relative cost saving under all the network conditions considered in the examples is between $10 \%-20 \%$, which translates to a large CAPEX ${ }^{3}$ saving considering the cost of LTEs.

\footnotetext{
${ }^{3}$ CAPEX refers to Capital Expenditure.
}

Almost the same cost saving ratio is achieved in the nondisjoint group case. The corresponding network cost factors are shown in the Table III. The case where groups may have common nodes results in further reduction in the overall cost compared to the case where each group has disjoint network nodes. However, the results demonstrates that the advantages of employing network coding are not affected.

\section{B. Un-hubbed unidirectional ring}

The network costs of un-hubbed unidirectional rings under various network scenarios are shown in Table IV with $g=4,8$ and 16. By inspecting the table, network cost saving increases as the total amount of traffic transmitted increases with the same $g$. However, the saving achieved by employing network coding decreases with the increase of the grooming factor, since the greater the grooming factor, the fewer lightpaths are set up for the given communication requests. Hence, the fewer lightpaths are saved by applying network coding, which results in less saving of LTEs.

Notice that when the number of nodes in each group is exactly 2 and the total number of nodes on the ring is 20 , the network cost is a constant regardless of the grooming factor and whether or not network coding is employed, because each node needs two LTEs, one as transmitter and one as receiver, to implement all-to-all communication without necessity of network coding and traffic grooming. Except for this case, the overall cost savings under other different network scenarios considered is between $1-5 \%$, which is less significant than the saving obtained by using network coding in single-hub rings.

In addition, we also obtain the network cost for the case of non-disjoint groups in the ring. As shown in Table V, the network cost for each network scenario is slightly less than its counterpart in the previous case where groups do not share any node, which illustrates the fact that sharing hub nodes among different groups does reduce the overall network 
TABLE IV

THE COMPARISON OF TOTAL NETWORK COST OF DISJOINT GROUPS IN THE UN-HUBBED RING WITH G=4,8 AND 16

\begin{tabular}{|c|c|c|c|c|c|c|c|c|c|c|c|c|c|}
\hline & (Traffic,nodes) & $(2,20)$ & $(2.5,20)$ & $(2,30)$ & $(2.5,30)$ & $(2,40)$ & $(2.5,40)$ & $(2,50)$ & $(2.5,50)$ & $(2,60)$ & $(2.5,60)$ & $(2,70)$ & $(2.5,70)$ \\
\hline \multirow[t]{2}{*}{$\mathrm{g}=4$} & Without NC & 40 & 40 & 81 & 84.3 & 145.2 & 154.1 & 212.5 & 233.7 & 300.3 & 337.1 & 405.7 & 450.8 \\
\hline & With NC & 40 & 40 & 80.1 & 83.5 & 138.8 & 149.6 & 207.2 & 229.7 & 290.6 & 329 & 396.6 & 443.7 \\
\hline \multirow[t]{2}{*}{$\mathrm{g}=8$} & Without NC & 40 & 40 & 72.4 & 73.8 & 117 & 121.3 & 159.3 & 170.7 & 218.3 & 236.7 & 285.4 & 307.6 \\
\hline & With NC & 40 & 40 & 71.2 & 72.2 & 113 & 117.3 & 156.5 & 167.1 & 209.5 & 228.8 & 276.8 & 300.6 \\
\hline \multirow[t]{2}{*}{$\mathrm{g}=16$} & Without NC & 40 & 40 & 69.1 & 69.1 & 103.7 & 106.6 & 136.8 & 142.1 & 176.9 & 186.1 & 225.4 & 235.2 \\
\hline & With NC & 40 & 40 & 69.1 & 69.1 & 100.8 & 102.7 & 134.9 & 139.7 & 172.5 & 180.9 & 219.7 & 230.3 \\
\hline
\end{tabular}

TABLE V

THE COMPARISON OF TOTAL NETWORK COST OF NON-DISJOINT GROUPS IN UN-HUBBED RING WITH G=8 AND 16

\begin{tabular}{|c|c|c|c|c|c|c|c|c|c|c|c|c|c|}
\hline & (Traffic,nodes) & $(2,20)$ & $(3,20)$ & $(2,30)$ & $(3,30)$ & $(2,40)$ & $(3,40)$ & $(2,50)$ & $(3,50)$ & $(2,60)$ & $(3,60)$ & $(2,70)$ & $(3,70)$ \\
\hline \multirow[t]{2}{*}{$\mathrm{g}=8$} & Without $\mathrm{NC}$ & 35.6 & 39.0 & 71.4 & 77.8 & 113.8 & 130.0 & 158.0 & 184.9 & 217.7 & 255.7 & 281.5 & 326.5 \\
\hline & With NC & 35.6 & 39.0 & 69.8 & 75.2 & 109.3 & 125.8 & 152.6 & 181.2 & 211.1 & 249.2 & 275.5 & 319.1 \\
\hline \multirow[t]{2}{*}{$g=16$} & Without NC & 35.4 & 36.4 & 65.1 & 68.4 & 100.5 & 105.4 & 132.6 & 143.3 & 171.2 & 190.1 & 214.6 & 240.4 \\
\hline & With NC & 35.4 & 36.4 & 64.3 & 66.5 & 97.5 & 101.4 & 130.1 & 140.1 & 167.3 & 185.0 & 210.2 & 234.5 \\
\hline
\end{tabular}

cost. However, the benefit that network coding could achieve remains almost the same as that in the disjoint group case.

The reason for the difference between single-hub rings and un-hubbed rings is that in an un-hubbed ring, all the traffic does not need to be transmitted to the same hub when using the multi-hub approach. Once a wavelength is filled up at a node, the data is broadcast. However, network coding requires a common hub on the ring to collect the data from all the nodes within the same group in the one-hub scheme. Even if a wavelength is fully loaded, it has to experience an extra delivery to the common hub. Such extra delivery consumes more LTEs in un-hubbed rings. Only if the number of nodes and traffic rate of a group satisfy certain conditions, can network coding save costs for this group. This means that not every group with an all-to-all traffic demand will benefit from network coding in un-hubbed rings. Therefore, the total saving - the sum of the saving from each group - will not be as high as that in single-hub rings.

\section{Vi. CONClusions}

In this paper we provided the first study of the traffic grooming problem of two types of unidirectional rings, singlehub and un-hubbed, with uniform all-to-all, and its extension, many-to-many, traffic scenarios, and with or without network coding. We considered the number of LTEs as the dominant factor of network cost. Traffic bifurcation and optical splitters are allowed in our analysis of network costs in all network scenarios.

Applying network coding into WDM ring networks introduces several issues: the first issue is the selection of coding nodes and coding coefficients and this has been addressed in our proposed schemes. Second, the encoding process may cause delay due to the synchronization between original traffic signals to be combined at the hubs. However, the typical endto-end delay in optical networks is very small due to the dedicated lightpaths provisioned for any end-to-end communication and thus network coding will not bring down the overall delay performance. Third, network coding introduces extra computation cost in application layer due to the encoding and decoding process performed in the electronic domain.
However, compared to the saving in CAPEX cost of a LTE, which reaches tens of thousands of dollars, the additional computation cost is much less and almost negligible.

In a single-hub ring, we explored the minimum cost of allto-all traffic in the cases when network coding was not applied and when it was applied, and from numerical results, we observed $10-20 \%$ cost savings with the deployment of network coding in two many-to-many communication scenarios, in which different groups are allowed and not allowed to have common nodes. In the un-hubbed unidirectional ring, we proposed the multi-hub scheme to derive the network cost when network coding is not applied and one-hub scheme if network coding is applied for many-to-many communication. We also consider a more general case where different communication groups are not node disjoint. An heuristic algorithm, HSM, is proposed to address this issue. We evaluated the network cost using this algorithm and compared the cost under the two cases where network coding is applied or not. Based on the numerical results for different network scenario, the savings of LTEs by employing network coding is $1-5 \%$, which is less significant than the saving in the single-hub ring case.

\section{REFERENCES}

[1] A. L. Chiu and E. H. Modiano, "Traffic grooming algorithms for reducing electronic multiplexing costs in WDM ring networks." IEEE/OSA Journal of Lightwave Technology, 18(1): 2-12, January 2000.

[2] J-C. Bermond and D. Coudert, "Traffic grooming in unidrirectional WDM ring networks: the all-to-all unitary case," in Proc. 7th IFIP Working Conference on Optical Network Design and Modeling, Feb. 2003.

[3] K. Zhu, B. Mukherjee, "Traffic grooming in an optical WDM mesh network," IEEE J. Select. Areas Commun. 20(1) (2002) 122-133.

[4] R. Dutta, G.N. Rouskas, "On optimal traffic grooming in WDM rings", IEEE J. Select. Areas Commun. 20:110-121, 2002.

[5] R. Ahlswede, N. Cai, S.-Y. R. Li, and R. W. Yeung, "Network information flow", IEEE Trans. Inf. Theory, vol. 46, pp. 1204-1216, July 2000.

[6] S.R.Li, R.W.Yeung, and N.Cai, "Linear network coding," IEEE Trans. Info. Theory, vol. 49, No. 2, pp.371-381, 2003.

[7] S. Jaggi, P. Sanders, P. A. Chou, M. Effros, S. Egner, K. Jain, and L. M. G. M. Tolhuizen, "Polynomial time algorithms for multicast network code construction," IEEE Trans. Inf. Theory, vol. 51, no. 6, pp. 19731982, 2005.

[8] T. Ho, M. Medard, R. Koetter, D.R. Karger, M. Effros, J. Shi and B. Leong, "A random linear network coding approach to multicast," IEEE Trans. Inform. Theory, vol. 52, no.10, pp. 4413C4430, 2006. 
[9] H. V. Madhyastha, G. V. Chowdhary, N. Srinivas, and C. S. R. Murthy, "Grooming of multicast sessions in metropolitan WDM ring networks," Computer Networks, vol. 49, no. 4, pp. 561-579, 2005.

[10] X. Y. Li, L. Liu, P. J. Wan, and O. Frieder, "Practical traffic grooming scheme for single-hub SONET/WDM rings," in Proc. 25th IEEE Conf. on Local Computer Networks, Nov. 2000.

[11] J. Q. Hu, "Optimal traffic grooming for WDM rings with all-to-all uniform traffic," Journal of Optical Networks, Vol. 1, 32-42, 2002.

[12] J. Wang, W. Cho, V. Vemuri and B. Mukherjee, "Improved approaches for cost-effective traffic grooming in WDM ring networks: ILP formulations and single-hop and multihop connections," Journal of Lightwave Technology, Vol. 19, No. 11, pp.1645-1653, 2001.

[13] G. Feng, C. Siew, and T-S. Yum, ”Architectural design and bandwidth demand analysis for multiparty videoconferenceing on SONET/ATM rings", IEEE J. Select. Areas Commu., vol. 20, no. 8, pp.1580-1588, 2002.

[14] Anuj Rawat, Richard La, Steven Marcus, and Mark Shayman, "Grooming Multicast Traffic in Unidirectional SONET/WDM Rings", IEEE J. Select. Areas Commun. 20(6) August, 2007.

[15] Kamal, A. E., "Algorithms for Multicast Traffic Grooming in WDM Mesh Networks", IEEE Communications, Volume 44, No. 11, Nov. 2006.

[16] Ul-Mustafa, R. and A. E. Kamal, "Many-to-one Traffic Grooming with Aggregation in WDM Networks" , IEEE Journal on Selected Areas in Communications, Part II: Optical Communications and Networking, Vol. 24, No. 8, Aug. 2006, pp. 68-81.

[17] Ul-Mustafa, R. and A. E. Kamal, "Design and Provisioning of WDM Networks with Multicast Traffic Grooming", IEEE Journal on Selected Areas in Communications, Part II: Optical Communications and Networking, Vol. 24, No. 4, Apr. 2006

[18] J.Q.Hu, Brett leida, "Traffic Grooming, Routing, and Wavelength Assignment in Optical WDM Mesh Networks," Proceedings of IEEE Infocom, 2004.

[19] X.Zhang and C.Qiao, "An effective and comprehensive approach for traffic grooming and wavelength assignment in SONET/WDM rings", IEEE/ACM Trans. Networking, vol. 8, no.5, pp. 608-617, Oct 2000.

[20] O. Gerstel, R. Ramaswami, and G. Sasaki, "Cost-effective traffic grooming in WDM rings", IEEE/ACM Trans. Networking, vol. 8, no.5, pp. 618630 , Oct 2000.

[21] P.-J. Wan, G.Galinescu, L.Liu and O.Frieder, "Grooming of arbitrary traffic in SONET/WDM BLSRs", IEEE Journal Select. Areas Commun., Vol. 18, no.10, pp.1995-2003, Oct.2000.

[22] M. Zhang, L. Wang and P. Ye, "All optical XOR logic gates: technologies and experiment demonstrations," IEEE Communications magazine, vol. 43, no.5, pp.s19-s24, May 2005.

[23] Eric D. Manley, Jitender S. Deogun and Lisong Xu, "Network coding for optical layer multicast," Proc. Broadnets 2008.

[24] T.Ho, B. Leong, Y. Chang, Y. Wen and R. Koetter, "Network monitoring in multicast networks using network coding", in International Symposium on Information Theory (ISIT) 2005.

[25] Kamal, A. E., "1+N Network Protection for Mesh Networks: Network Coding-Based Protection using p-Cycles", to appear in IEEE/ACM Transactions on Networking.

[26] Al-Kofahi, O. and A. E. Kamal, "Network Coding-Based Protection of Many-to-One Wireless Flows", to appear in IEEE J. Select. Areas Commun. special issue on Network Coding in Wireless Commun., 2009.

[27] C. Gkantsidis and P. Rodriguez, "Network coding for large scale content distribution", Proceedings of IEEE Infocom, 2005.

[28] S. Katti, H. Rahul, W. Hu, D. Katabi, M. Medard and J. Crowcroft, "XORs in the Air: Practical Wireless Network Coding", in Proceedings of SIGCOMM 2006.

[29] E.G. Coffman, M.R. Garey, D.S. Johnson, "Approximation algorithms for bin packing - A survey. In: Approximation Algorithms for NP-Hard Problems", PWS Publishing Company, Boston, pp 46-93, 1997.

[30] J.M.V de Carvalho, "Exact solution of bin packing problems using column generation and branch and bound ", Annals of Operations Research, Springer, Volume 86, No. 0, January, 1999
Ahmed E. Kamal (S'82-M'87-SM'91) received a B.Sc. (distinction with honors) and an M.Sc. both from Cairo University, Egypt, and a M.A.Sc. and a Ph.D. both from the University of Toronto, Canada, all in Electrical Engineering in 1978, 1980, 1982 and 1986, respectively. He is currently a professor of Electrical and Computer Engineering at Iowa State University. Earlier he held faculty positions in the Department of Computing Science at the University of Alberta, Canada, and the Department of Computer Engineering at Kuwait University, Kuwait. He was also an adjunct professor at the Telecommunications Research Labs, Edmonton, Alberta. Kamals research interests include high-performance networks, optical networks, wireless and sensor networks and performance evaluation. He is a student member of IEEE.

$\mathrm{He}$ is a senior member of the IEEE, a member of the Association of Computing Machinery, and a registered professional engineer. He was the corecipient of the 1993 IEE Hartree Premium for papers published in Computers and Control in IEE Proceedings for his paper entitled Study of the Behaviour of Hubnet, and the best paper award of the IEEE Globecom 2008 Symposium on Ad Hoc and Sensors Networks Symposium. He served on the technical program committees of numerous conferences and workshops, was the organizer and co-chair of the first and second Workshops on Traffic Grooming 2004 and 2005, respectively, and was the co-chair of the Technical Program Committees of a number of conferences including the Communications Services Research (CNSR) conference 2006, the Optical Symposium of Broadnets 2006, and the Optical Networks and Systems Symposium of the IEEE Globecom 2007, the 2008 ACS/IEEE International Conference on Computer Systems and Applications (AICCSA-08), and the ACM International Conference on Information Science, Technology and Applications, 2009. He is also the Technical Program co-chair of the Optical Networks and Systems Symposium of the IEEE Globecom 2010. He is on the editorial boards of the Computer Networks journal, and the Journal of Communications.
Long Long received his B.Eng degree in Electronic Information Engineering from Huazhong University of Science and Technology, Wuhan, China in 2002 and M.Sc degree in Software Engineering from Peking University, Beijing, China in 2005. Since fall 2006, he has been a Ph.D student in ECE department of Iowa State University, USA. His research interests are in the area of traffic grooming and survivability of optical networks. 